\title{
ОДНОМЕРНЫЕ ВОЛНОВЫЕ УРАВНЕНИЯ, РЕШАЕМЫЕ В ЗАМКНУТОМ ВИДЕ
}

\section{Введение}

В предыдущих статьях $\left[{ }^{1-7}\right]$ был развит в нескольких формах метод, позволяющий находить одномерные волновые уравнения и их решения в замкнутом виде на базе уже известных решений дифференциальных уравнений второго порядка. Там же был приведен ряд примеров. В настоящей статье рассмотрим несколько новых примеров, ранее в [ $\left.{ }^{1}\right]$ только в самых общих чертах намеченных. Воспользуемся данной в $\left[{ }^{6}\right]$ формой нашего метода, начав с его краткого изложения в несколько улучшенном виде.

\section{Метод}

Каждое дифференциальное уравнение второго порядка вида

$$
d^{2} y / d x^{2}+f(x) y=0
$$

может быть преобразовано в уравнение такого же вида подстановкой

$$
\begin{aligned}
& x=x(\xi), \\
& y=(d x / d \xi)^{1 / 2} \eta,
\end{aligned}
$$

где $x(\xi)$ - произвольная трижды дифференцируемая функция. В самом деле, выполнив все действия, находим

$$
d^{2} \eta / d \xi^{2}+\varphi(\xi) \eta=0,
$$

где

$$
\varphi(\xi)=f(x(\xi))(d x / d \xi)^{2}-\frac{3}{4}\left(\frac{d^{2} x / d \xi^{2}}{d x / d \xi}\right)^{2}+\frac{1}{2} \cdot \frac{d^{3} x / d \xi^{3}}{d x / d \xi} .
$$

Очевидно, преобразования (2) образуют группу.

В нашем методе вывода новых волновых уравнений преобразования вида (2) находят двоякое применение. Одно из них состоит в придании более общей формы какому-либо уже найденному волновому уравнению. С этой целью уравнение преобразуется по формуле (2), в которой новый аргумент берется в виде дробно-линейной функции старого. Эти преобразования составляют, очевидно, подгруппу группы всех преобразований вида (2). Итак, пусть имеем волновое уравнение

$$
d^{2} U / d z^{2}+k^{2} n^{2}(z) U=0,
$$

где $k$ - волновое число, $n(z)$ - зависящий от координаты $z$ показатель преломления. Преобразование

$$
z / h \rightarrow \frac{a z / h+b}{c z / h+d}
$$

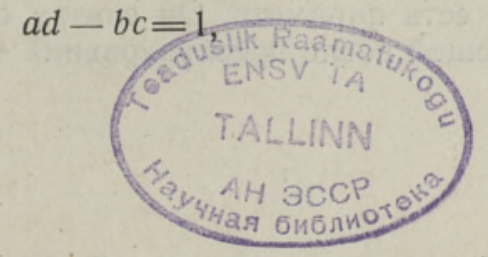




$$
U \rightarrow(c z / h+d)^{-1} U
$$

где $h$ - постоянная размерности длины (обычно толщина слоя), превращает это уравнение в более общее уравнение

$$
d^{2} U / d z^{2}+k^{2}(c z / h+d)^{-4} n^{2}\left(\frac{a z / h+b}{c z / h+d}\right) U=0 .
$$

Если решение уравнения (5) известно, то через него, согласно второй формуле (6), выражается и решение уравнения (7). Понятно, повторное преобразование вида (6) не приводит к еще большей общности, а означает только замену чисел $a, b, c, d$ другими. В нижеследующем будем каждый раз при выводе нового волнового уравнения пользоваться преобразованием вида (6) без особой оговорки.

Другое применение преобразования (2) составляет ядро нашего метода. Исходным уравнением является уравнение, имеющее известное решение, вида

$$
d^{2} \theta / d G^{2}+\left[k^{2} h^{2} N^{2}(G)+q(G)\right] \theta=0,
$$

где $N(G)$ и $q(G)$ - заданные функции.. Если положим

$$
\begin{aligned}
& G=G(z / h), \\
& \theta=(h d G / d z)^{1 / 2} U,
\end{aligned}
$$

то для $U(z)$ получим уравнение

$$
\begin{gathered}
d^{2} U / d z^{2}+\left\{\left[k^{2} h^{2} N^{2}(G)+q(G)\right](d G / d z)^{2}-\right. \\
\left.-\frac{3}{4}\left(\frac{d^{2} G / d z^{2}}{d G / d z}\right)^{2}+\frac{1}{2} \cdot \frac{d^{3} G / d z^{3}}{d G / d z}\right\} U=0
\end{gathered}
$$

Чтобы это уравнение было волновым, нужно, чтобы не зависящая от $k$ часть обращалась в нуль:

$$
q(G)(d G / d z)^{2}-\frac{3}{4}\left(\frac{d^{2} G / d z^{2}}{d G / d z}\right)^{2}+\frac{1}{2} \cdot \frac{d^{3} G / d z^{3}}{d G / d z}=0
$$

Это условие подстановкой

$$
d G / d z=h^{-1} \Psi^{2}
$$

приводится к линейному уравнению второго порядка

$$
d^{2} \Psi / d G^{2}+q(G) \Psi=0
$$

линейно независимые решения $\Psi_{1}$ и $\Psi_{2}$ которого (или хотя бы одно из них) получаются как $\lim _{h \rightarrow 0} \theta(G)$. Пусть

$$
\Psi_{1} d \Psi_{2} / d G-\Psi_{2} d \Psi_{1} / d G=1
$$

и в уравнении (12) $\Psi=\Psi_{1}$. Интегрируя это уравнение, находим

$$
\Psi_{2}(G) / \Psi_{1}(G)=z / h .
$$

Волновое уравнение (10), в силу равенств (11) и (12) и с учетом преобразования (6), получается в виде

$$
d^{2} U / d z^{2}+k^{2}(c z / h+d)^{-4} N^{2}(G) \Psi_{1}^{4}(G) U=0,
$$

где $G$ есть параметр. Он связан с координатой $z$ формулой $(15)$, принимающей после преобразования (6) вид 


$$
\Psi_{2}(G) / \Psi_{1}(G)=\frac{a z / h+b}{c z / h+d} .
$$

Решение волнового уравнения (16), в силу формул (9) и (12) и преобразования (6), есть

$$
U=(c z / h+d) \Psi_{1}^{-1} \theta .
$$

Итак, каждому уравнению вида (8) соответствует волновое уравнение вида (16), решаемое в замкнутом виде, если известно решение уравнения (8). Часто решение остается в параметрическом виде, но иногда параметр поддается исключению. Отметим, что выбор линейно независимых функций $\Psi_{1}$ и $\Psi_{2}$ произволен, поскольку переход $\mathrm{K}$ их линейным комбинациям эквивалентен, очевидно, преобразованию (6). нениях

Переходим к примерам. Все они в данной статье основаны на урав-

$$
\begin{aligned}
& d^{2} \theta / d G^{2}+\left(k^{2} h^{2} N_{0}^{2}-C^{2}-2 \operatorname{sh}^{-2} G\right) \theta=0 \\
& d^{2} \theta / d G^{2}+\left(k^{2} h^{2} N_{0}^{2}+C^{2}-2 \sin ^{-2} G\right) \theta=0 \\
& d^{2} \theta / d G^{2}+\left(k^{2} h^{2} N_{0}^{2}+C^{2}-2 \operatorname{sh}^{-2} G\right) \theta=0
\end{aligned}
$$

и

$$
d^{2} \theta / d G^{2}+\left(k^{2} h^{2} N_{0}^{2}-C^{2}-2 \sin ^{-2} G\right) \theta=0,
$$

где $N_{0}$ и $C$ - постоянные. В зависимости от значения $C$ отсюда получается множество конкретных волновых уравнений. Целесообразно рассмотреть сначала, особо простейшие варианты, а потом более сложные.

\section{Простейшие примеры}

I. Если в уравнении (19) или (21) $C=0$, то

$$
d^{2} \theta / d G^{2}+\left(k^{2} h^{2} N_{0}^{2}-2 \operatorname{sh}^{-2} G\right) \theta=0 .
$$

Решение этого уравнения есть

$$
\theta=\left(\text { cth } G \mp i k h N_{0}\right) \exp \left( \pm i k h N_{0} G\right) \text {. }
$$

Для $\Psi$ имеем уравнение

$$
d^{2} \Psi / d G^{2}-2 \operatorname{sh}^{-2} G \cdot \Psi=0,
$$

линейно независимые решения которого выберем в виде

$$
\begin{aligned}
& \Psi_{1}=\operatorname{cth} G, \\
& \Psi_{2}=G \operatorname{cth} G-1,
\end{aligned}
$$

так что

$$
G-\text { th } G=\frac{a z / h+b}{c z / h+d} .
$$

Волновое уравнение получается в виде

$$
d^{2} U / d z^{2}+k^{2} N_{0}^{2}(c z / h+d)^{-4} \operatorname{cth}^{4} G \cdot U=0 .
$$

Решение его есть

$$
U=(c z / h+d)\left(1 \mp i k h N_{0} \text { th } G\right) \exp \left( \pm i k h N_{0} G\right)
$$


II. Если в уравнении (20) или (22) $C=0$, то

$$
d^{2} \theta / d G^{2}+\left(k^{2} h^{2} N_{0}^{2}-2 \sin ^{-2} G\right) \theta=0 .
$$

Аналогично предыдущему случаю находим волновое уравнение

$$
d^{2} U / d z^{2}+k^{2} N_{0}^{2}(c z / h+d)^{-4} \operatorname{ctg}^{4} G \cdot U=0,
$$

где

$$
\tan G-G=\frac{a z / h+b}{c z / h+d}
$$

Решение уравнения (31) есть

$$
U=(c z / h+d)\left(1 \mp i k h N_{0} \tan G\right) \exp \left( \pm i k h N_{0} G\right) .
$$

III. Если в уравнении (19) положим $C=1$, то

$$
d^{2} \theta / d G^{2}+\left(k^{2} h^{2} N_{0}^{2}-1-2 \operatorname{sh}^{-2} G\right) \theta=0
$$

и

$$
d^{2} \Psi / d G^{2}-\left(1+2 \mathrm{sh}^{-2} G\right) \Psi=0 .
$$

Эти уравнения имеют решения

$$
\theta=\left(\operatorname{cth} G \mp i \sqrt{k^{2} h^{2} N_{0}^{2}-1}\right) \exp \left( \pm i G \sqrt{k^{2} h^{2} N_{0}^{2}-1}\right)
$$

и

$$
\begin{aligned}
& \Psi_{1}=2^{-1 / 2} \operatorname{sh}^{-1} G, \\
& \Psi_{2}=2^{-1 / 2}\left(\operatorname{ch} G-G \operatorname{sh}^{-1} G\right) .
\end{aligned}
$$

Отсюда находим волновое уравнение

$$
d^{2} U / d z^{2}+\frac{1}{4} k^{2} N_{0}^{2}(c z / h+d)^{-4} \mathrm{sh}^{-4} G \cdot U=0
$$

с решением

$$
U=(c z / h+d)\left(\operatorname{ch} G \mp i \operatorname{sh} G \sqrt{k^{2} h^{2} N_{0}^{2}-1}\right) \exp \left( \pm i G \sqrt{k^{2} h^{2} N_{0}^{2}-1}\right),
$$

причем

$$
\operatorname{sh} G \operatorname{ch} G-G=\frac{a z / h+b}{c z / h+d} .
$$

IV. Если в уравнении (20) возьмем $C=1$, то, аналогично предыдущему случаю, находим волновое уравнение

$$
d^{2} U / d z^{2}+\frac{1}{4} k^{2} N_{0}^{2}(c z / h+d)^{-4} \sin ^{-4} G \cdot U=0
$$

с решением

$$
U=(c z / h+d)\left(\cos G \mp i \sin G \sqrt{k^{2} h^{2} N_{0}^{2}+1}\right) \exp \left( \pm i G \sqrt{k^{2} h^{2} N_{0}^{2}+1}\right),
$$
причем

$$
G-\sin G \cos G=\frac{a z / h+b}{c z / h+d} .
$$

В рассмотренных четырех примерах параметр $G$ не поддается исключению, хотя препятствием служит в сущности линь отсутствие общепринятых обозначений для функций, обратных функциям $G$-th $G$, 
$\tan G-G, \operatorname{sh} G-G$ и $G-\sin G$. Оставляя вопрос о целесообразности введения таковых обозначений открытым, перейдем к более сложным примерам. В некоторых из них параметр исключается.

\section{Более сложные примеры}

V. Пусть в уравнении (21) $C$ имеет произвольное отличное от нуля значение. Решение этого уравнения есть

$$
\theta=\left(\operatorname{cth} G \mp i \sqrt{k^{2} h^{2} N_{0}^{2}+C^{2}}\right) \exp \left( \pm i G \sqrt{k^{2} h^{2} N_{0}^{2}+C^{2}}\right),
$$

а решения уравнения

$$
d^{2} \Psi / d G^{2}+\left(C^{2}-2 \operatorname{sh}^{-2} G\right) \Psi=0
$$

возьмем в виде

$$
\begin{aligned}
& \Psi_{1}=\frac{C \cos C G-\sin C G \operatorname{cth} G}{\sqrt{C\left(C^{2}+1\right)}} \\
& \Psi_{2}=\frac{C \sin C G+\cos C G \operatorname{cth} G}{\sqrt{C\left(C^{2}+1\right)}} .
\end{aligned}
$$

Тогда получим волновое уравнение

$$
\frac{d^{2} U}{d z^{2}}+\frac{k^{2} N_{0}^{2}(c z / h+d)^{-4}(C \cos C G-\sin C G \text { cth } G)^{4} U}{C^{2}\left(C^{2}+1\right)^{2}}=0,
$$

решение которого есть

$$
U=\frac{(c z / h+d)\left(\operatorname{cth} G \mp i \sqrt{k^{2} h^{2} N_{0}^{2}+C^{2}}\right) \exp \left( \pm i G \sqrt{k^{2} h^{2} N^{2}+C^{2}}\right)}{C \cos C G-\sin C G \operatorname{cth} G}
$$

причем

$$
\frac{1+C \operatorname{th} G \tan C G}{C \operatorname{th} G-\tan C G}=\frac{a z / h+b}{c z / h+d} .
$$

VI. Если в уравнении (22) $C$ имеет произвольное отличное от нуля значение, то, аналогично предыдущему случаю, получаем волновое уравнение

$$
\frac{d^{2} U}{d z^{2}}+\frac{k^{2} N_{0}^{2}(c z / h+d)^{-4}(C \operatorname{ch} C G-\operatorname{sh} C G \operatorname{ctg} G)^{4} U}{C^{2}\left(C^{2}+1\right)^{2}}=0
$$

с решением

$$
U=\frac{(c z / h+d)\left(\operatorname{ctg} G \mp i \sqrt{k^{2} h^{2} N_{0}^{2}-C^{2}}\right) \exp \left( \pm i G \sqrt{k^{2} h^{2} N_{0}^{2}-C^{2}}\right)}{C \operatorname{ch} C G-\operatorname{sh} C G \operatorname{ctg} G}
$$

причем

$$
\frac{C \operatorname{th} C G \tan G-1}{C \tan G-\operatorname{th} C G}=\frac{a z / h+b}{c z / h+d}
$$

VII. Положим в уравнении (19) $C \neq 0, C \neq 1$, а в остальном пусть $C$ произвольно. Тогда решение его имеет вид 


$$
\theta=\left(\operatorname{cth} G \mp i \sqrt{k^{2} h^{2} N_{0}^{2}-C^{2}}\right) \exp \left( \pm i G \sqrt{k^{2} h^{2} N_{0}^{2}-C^{2}}\right) .
$$

Для $\Psi$ имеем уравнение

$$
d^{2} \Psi / d G^{2}-\left(C^{2}+2 \operatorname{sh}^{-2} G\right) \Psi=0,
$$

решения которого возьмем в виде

$$
\begin{aligned}
& \Psi_{1}=\frac{C \operatorname{ch} C G-\operatorname{sh} C G \operatorname{cth} G}{\sqrt{C\left(C^{2}-1\right)}}, \\
& \Psi_{2}=\frac{C \operatorname{sh} C G-\operatorname{ch} C G \operatorname{cth} G}{\sqrt{C\left(C^{2}-1\right)}} .
\end{aligned}
$$

Отсюда находим

$$
\frac{1-C \text { th } G \text { th } C G}{\text { th } C G-C \text { th } G}=\frac{a z / h+b}{c z / h+d} \text {. }
$$

Волновое уравнение получается в виде

$$
\frac{d^{2} U}{d z^{2}}+\frac{k^{2} N_{0}^{2}(c z / h+d)^{-4}(C \operatorname{ch} C G-\operatorname{sh} C G \operatorname{cth} G)^{4} U}{C^{2}\left(C^{2}-1\right)^{2}}=0,
$$

a решение его есть

$$
U=\frac{(c z / h+d)\left(\operatorname{cth} G \mp i \sqrt{k^{2} h^{2} N_{0}^{2}-C^{2}}\right) \exp \left( \pm i G \sqrt{k^{2} h^{2} N_{0}^{2}-C^{2}}\right)}{C \operatorname{ch} C G-\operatorname{sh} C G \operatorname{cth} G} .
$$

VIII. Аналогично предыдущему случаю положим в уравнении (20) $C \neq 0, C \neq 1$, а в остальном пусть $C$ произвольно. Тогда

$$
\theta=\left(\operatorname{ctg} G \mp i \sqrt{k^{2} h^{2} N_{0}^{2}+C^{2}}\right) \exp \left( \pm i G \sqrt{k^{2} h^{2} N_{0}^{2}+C^{2}}\right) .
$$

Для $\Psi$ имеем уравнение

$$
d^{2} \Psi / d G^{2}+\left(C^{2}-2 \sin ^{-2} G\right) \Psi=0
$$

c решениями

$$
\begin{aligned}
& \Psi_{1}=\frac{C \cos C G-\sin C^{\prime} \operatorname{ctg} G}{\sqrt{C\left(C^{2}-1\right)}} \\
& \Psi_{2}=\frac{C \sin C G+\cos C G \operatorname{ctg} G}{\sqrt{C\left(C^{2}-1\right)}}
\end{aligned}
$$

Отсюда

$$
\frac{1+C \tan C G \tan G}{C \tan G-\tan C G}=\frac{a z / h+b}{c z / h+d} .
$$

Волновое уравнение имеет вид

$$
\frac{d^{2} U}{d z^{2}}+\frac{k^{2} N_{0}^{2}(c z / h+d)^{-4}(C \cos C G-\sin C G \operatorname{ctg} G)^{4} U}{C^{2}\left(C^{2}-1\right)^{2}}=0
$$

а решение его есть 


$$
U=\frac{(c z / h+d)\left(\operatorname{ctg} G \mp i \sqrt{k^{2} h^{2} N_{0}^{2}+C^{2}}\right) \exp \left( \pm i G \sqrt{k^{2} h^{2} N_{0}^{2}+C^{2}}\right)}{C \cos C G-\sin C G \operatorname{ctg} G}
$$

В рассмотренных примерах V-VIII параметр, кроме некоторых случаев, не поддается исключению. Нет большого смысла и вводить особые обозначения для обратных функций, поскольку зависимость координаты от параметра здесь не столь проста.

B примерах VII и VIII параметр при отдельных значениях $C$ всетаки исключается. Рассмотрим некоторые из этих случаев особо.

\section{Случаи с исключенным параметром}

В примерах VII и VIII параметр, вообще говоря, исключается при значениях C $1 / 2,2,1 / 3$ и 3 , хотя в некоторых случаях приходится вводить другой параметр. Обозначив для краткости

$$
\xi=\frac{a z / h+b}{c z / h+d},
$$

получаем из формулы (56) при этих значениях $C$ следующие уравнения 3 -й и 4 -й степени

$$
\begin{aligned}
& \operatorname{cth}^{3}(G / 2)-\xi=0, \\
& \operatorname{cth}^{3} G-3 \operatorname{cth} G+2 \xi=0, \\
& 3 \operatorname{cth}^{4}(G / 3)+6 \operatorname{cth}^{2}(G / 3)-8 \xi \operatorname{cth}(G / 3)-1=0
\end{aligned}
$$

и

$$
\operatorname{cth}^{4} G-6 \text { cth }^{2} G+8 \xi \text { cth } G-3=0 .
$$

Аналогично, формула (62) дает:

$$
\begin{aligned}
& \operatorname{ctg}^{3}(G / 2)-\xi=0, \\
& \operatorname{ctg}^{3} G+3 \operatorname{ctg} G+2 \xi=0, \\
& 3 \operatorname{ctg}^{4}(G / 3)-6 \operatorname{ctg}^{2}(G / 3)-8 \xi \operatorname{ctg}(G / 3)-1=0
\end{aligned}
$$

и

$$
\operatorname{ctg}^{4} G+6 \operatorname{ctg}^{2} G+8 \xi \operatorname{ctg} G-3=0 .
$$

Все эти уравнения решаются в радикалах и, следовательно, параметр $G$ может быть исключен (правда, в некоторых случаях неизбежно введение другого параметра, о чем см. ниже). При других значениях $C$ параметр не поддается исключению; при $C=m$ или $C=m^{-1}$, где $m>3$ целое число, для $\operatorname{cth} G$ и $\operatorname{ctg} G$ или $\operatorname{cth}(G / m)$ и $\operatorname{ctg}(G / m)$ получаются уравнения $(m+1)$-й степени, не решаемые в радикалах. Если же ни $C$, ни $C^{-1}$ не целое, то уравнения (56) и (62) оказываются трансцендентными.

В настоящей статье мы не будем рассматривать значений $C=3$ и $C=1 / 3$, приводящих к волновым уравнениям очень громоздкого вида. Оставим в стороне также случаи с $C=1 / 2$, в которых волновые уравнения совпадают с найденными несколько иным путем ранее (см. $\left[{ }^{7}\right]$, уравнения (55) и (65)). Различие обнаруживается только в том, что в $\left[{ }^{7}\right]$ роль величины $\frac{a z / h+b}{c z / h+d}$ играет $\frac{B(a z / h+b)}{c z / h+d}$. Это различие, однако, несущественно, так как $\frac{B(a z / h+b)}{c z / h+d}=\frac{B^{1 / 2} a z / h+B^{1 / 2} b}{B^{-1 / 2} c z / h+B^{-1 / 2} d}$; 
мена $B^{1 / 2} a \rightarrow a, B^{1 / 2} b \rightarrow b ; B^{-1 / 2} c \rightarrow c, B^{-1 / 2} d \rightarrow d$ устраняет $B$, сохраняя условие $a d-b c=1$.

Остается рассмотреть случаи с $C=2$.

1. Относящееся к VII примеру с $C=2$ уравнение (67) в случае $|\xi|>1$ решается по формуле Кардана

$$
\text { cth } G=-\left(\xi+\sqrt{\xi^{2}-1}\right)^{1 / 3}-\left(\xi-\sqrt{\xi^{2}-1}\right)^{1 / 3} .
$$

Волновое уравнение (57) при $C=2$ получается в виде

$$
\frac{d^{2} U}{d z^{2}}+\frac{4 k^{2} N_{0}^{2}(c z / h+d)^{-4} U}{9\left(\operatorname{cth}^{2} G-1\right)^{4}}=0 .
$$

Подставляя сюда вместо cth $G$ выражение (74) и заменяя $N_{0} \rightarrow 6 N_{0}$, находим

$$
\begin{gathered}
d^{2} U / d z^{2}+k^{2} N_{0}^{2}\left[\left(a^{2}-c^{2}\right) z^{2} / h^{2}+2(a b-c d) z / h+\left(b^{2}-d^{2}\right)\right]^{-2} \times \\
\times\left[\left(\xi+\sqrt{\xi^{2}-1}\right)^{1 / 3}-\left(\xi-\sqrt{\xi^{2}-1}\right)^{1 / 3}\right]^{4} U=0,
\end{gathered}
$$

где для сокращения записи оставлено छ вместо $\frac{a z / h+b}{c z / h+d}$ (см. формулу (65)). Решение этого уравнения дается формулой (58), принимающей вид

$$
\begin{aligned}
U= & {\left[\left(a^{2}-c^{2}\right) z^{2} / h^{2}+2(a b-c d) z / h+\left(b^{2}-d^{2}\right)\right]^{1 / 2} \times } \\
& \times \frac{\left(\xi+\sqrt{\xi^{2}-1}\right)^{1 / 3}+\left(\xi-\sqrt{\xi^{2}-1}\right)^{1 / 3} \pm 2 i \sqrt{9 k^{2} h^{2} N_{0}^{2}-1}}{\left(\xi+\sqrt{\xi^{2}-1}\right)^{1 / 3}-\left(\xi-\sqrt{\xi^{2}-1}\right)^{1 / 3}} \times \\
& \times \exp \left( \pm i \sqrt{9 k^{2} h^{2} N_{0}^{2}-1} \ln \frac{\left(\xi+\sqrt{\xi^{2}-1}\right)^{1 / 3}+\left(\xi-\sqrt{\xi^{2}-1}\right)^{1 / 3}-1}{\left(\xi+\sqrt{\xi^{2}-1}\right)^{1 / 3}+\left(\xi-\sqrt{\xi^{2}-1}\right)^{1 / 3}+1}\right) .
\end{aligned}
$$

2. Продолжая VII пример с $C=2$ положим $|\xi|<1$. Тогда уравнение (67) имеет три вещественных корня и формула Кардана непосредственно неприменима. Поэтому мы должны вновь ввести параметр, причем трем корням уравнения (67) соответствуют три различных волновых уравнения.

Положим

$$
\cos \omega=\xi=\frac{a z / h+b}{c z / h+d},
$$

где $\dot{\omega}-$ новый параметр. Тогда

$$
\begin{aligned}
& \text { cth } G_{1}=-2 \cos (\omega / 3), \\
& \text { cth } G_{2}=-2 \cos (\omega / 3+2 \pi / 3), \\
& \text { cth } G_{3}=-2 \cos (\omega / 3-2 \pi / 3) .
\end{aligned}
$$

Подставляя эти значения в уравнение (75), находим следующие три волновых уравнения (после замены $N_{0} \rightarrow 6 N_{0}$ ):

$$
\begin{aligned}
& \frac{d^{2} U}{d z^{2}}+\frac{16 k^{2} N_{0}^{2}(c z / h+d)^{-4} U}{[1+2 \cos (2 \omega / 3)]^{4}}=0, \\
& \frac{d^{2} U}{d z^{2}}+\frac{16 k^{2} N_{0}^{2}(c z / h+d)^{-4} U}{\left[1-\cos (2 \omega / 3)+3^{1 / 2} \sin (2 \omega / 3)\right]^{4}}=0
\end{aligned}
$$




$$
\frac{d^{2} U}{d z^{2}}+\frac{16 k^{2} N_{0}^{2}(c z / h+d)^{-4} U}{\left[1-\cos (2 \omega / 3)-3^{1 / 2} \sin (2 \omega / 3)\right]^{4}}=0
$$

Решения их, согласно формуле (58), получаются, соответственно, та́кими:

$$
\begin{aligned}
U= & (c z / h+d)[1+2 \cos (2 \omega / 3)]\left[\cos (\omega / 3) \pm i \sqrt{9 k^{2} h^{2} N_{0}^{2}-1}\right] \times \\
& \times \exp \left( \pm i \sqrt{9 k^{2} h^{2} N_{0}^{2}-1} \ln \frac{2 \cos (\omega / 3)-1}{2 \cos (\omega / 3)+1}\right) \\
U= & (c z / h+d)\left[1-\cos (2 \omega / 3)+3^{1 / 2} \sin (2 \omega / 3)\right] \times \\
& \times\left[\cos (\omega / 3)+3^{1 / 2} \sin (\omega / 3) \mp 2 i \sqrt{9 k^{2} h^{2} N_{0}^{2}-1}\right] \times \\
& \times \exp \left( \pm i \sqrt{9 k^{2} h^{2} N_{0}^{2}-1} \ln \frac{\cos (\omega / 3)+3^{1 / 2} \sin (\omega / 3)+1}{\cos (\omega / 3)+3^{1 / 2} \sin (\omega / 3)-1}\right)
\end{aligned}
$$

и

$$
\begin{aligned}
U= & (c z / h+d)\left[1-\cos (2 \omega / 3)-3^{1 / 2} \sin (2 \omega / 3)\right] \times \\
& \times\left[\cos (\omega / 3)-3^{1 / 2} \sin (\omega / 3) \mp 2 i \sqrt{9 k^{2} h^{2} N_{0}^{2}-1}\right] \times \\
& \times \exp \left( \pm i \sqrt{9 k^{2} h^{2} N_{0}^{2}-1} \ln \frac{\cos (\omega / 3)-3^{1 / 2} \sin (\omega / 3)+1}{\cos (\omega / 3)-3^{1 / 2} \sin (\omega / 3)-1}\right) .
\end{aligned}
$$

Здесь следует отметить следующие два обстоятельства. Во-первых, так как произведение трех значений cth $G$ равно $-2 \xi$ (см. формулы (79) или уравнение (67)) и $|\xi|<1$, то по крайней мере одно из этих значений по абсолютной величине меньше единищы. Это означает комплексность $G$. Однако, поскольку этот параметр исключен, его комплексность в уравнениях $(80)-(82)$ никакой роли не играет. Новый параметр $\omega$ в любом случае, согласно формуле (78), веществен.

Другое замечание состоит в следующем. Возможен случай, когда величина $\left|\frac{a z / h+b}{c z / h+d}\right|$ в одной и той же задаче переходит границу 1. Тогда уравнения (76) и (80) следует рассматривать как две переходящие друг в друга формы одного и того же уравнения. На границе, т. е. при $|\xi|=1$, коэффициент при $U$ равен в обоих уравнениях $(16 / 81) k^{2} N_{0}^{2}(a-\operatorname{sgn} \xi \cdot c)^{4}$, а решения (77) и (83) сшиваются, поскольку на границе совпадают значения как их самих

$$
\begin{aligned}
U_{|\xi|=1}= & 3(a-\operatorname{sgn} \xi \cdot c)^{-1}\left(\operatorname{sgn} \xi \pm i \sqrt{9 k^{2} h^{2} N_{0}^{2}-1}\right) \times \\
& \times \exp \left(\mp i \operatorname{sgn} \xi \sqrt{9 k^{2} h^{2} N_{0}^{2}-1} \cdot \ln 3\right)
\end{aligned}
$$

так и их первых производных

$$
\begin{aligned}
& (d U / d z)_{|\xi|=1}=h^{-1}\left[3 c \left(\operatorname{sgn} \xi \pm i \sqrt{9 k^{2} h^{2} N_{0}^{2}-1}+\right.\right. \\
& \left.+(1 / 3)(a-\operatorname{sgn} \xi \cdot c)\left(5 \pm 4 i \operatorname{sgn} \xi \sqrt{9 k^{2} h^{2} N_{0}^{2}-1}-12 k^{2} h^{2} N_{0}^{2}\right)\right] \times \\
& \times \exp \left(\mp i \operatorname{sgn} \xi \sqrt{9 k^{2} h^{2} N_{0}^{2}-1} \cdot \ln 3\right) .
\end{aligned}
$$

3. Рассмотрим, наконец, VIII пример с $C=2$. Относящееся к этому случаю уравнение (71) имеет единственный вещественный корень 


$$
\operatorname{ctg} G=\left(-\xi+\sqrt{\xi^{2}+1}\right)^{1 / 3}-\left(\xi+\sqrt{\xi^{2}+1}\right)^{1 / 3} .
$$

Подставляя это выражение в уравнение (63) и заменяя $N_{0} \rightarrow 6 N_{0}$, находим

$$
\begin{gathered}
d^{2} U / d z^{2}+k^{2} N_{0}^{2}\left[\left(a^{2}+c^{2}\right) z^{2} / h^{2}+2(a b+c d) z / h+\left(b^{2}+d^{2}\right)\right]^{-2} \times \\
+\left[\left(\xi+\sqrt{\xi^{2}+1}\right)^{1 / 3}+\left(-\xi+\sqrt{\xi^{2}+1}\right)^{1 / 3}\right]^{4} U=0 .
\end{gathered}
$$

Решение этого волнового уравнения получаем из формулы (64)

в виде

$$
\begin{aligned}
U= & {\left[\left(a^{2}+c^{2}\right) z^{2} / h^{2}+2(a b+c d) z / h+\left(b^{2}+d^{2}\right)\right]^{1 / 2} \times } \\
& \times \frac{\left(\xi+\sqrt{\xi^{2}+1}\right)^{1 / 3}-\left(-\xi+\sqrt{\xi^{2}+1}\right)^{1 / 3} \mp 2 i \sqrt{9 k^{2} h^{2} N_{0}^{2}+1}}{\left(\xi+\sqrt{\xi^{2}+1}\right)^{1 / 3}+\left(-\xi+\sqrt{\xi^{2}+1}\right)^{1 / 3}} \times \\
& \times \exp \left( \pm 2 i \sqrt{9 k^{2} h^{2} N_{0}^{2}+1} \operatorname{arcctg}\left[\left(\xi+\sqrt{\xi^{2}+1}\right)^{1 / 3}-\left(-\xi+\sqrt{\xi^{2}+1}\right)^{1 / 3} j\right) .\right.
\end{aligned}
$$

\section{Л И ТЕ Р А Т У Р А}

1. Кард П. Изв. АН ЭССР, Физ. Матем., 26, № 3, 252-259 (1977).

2. Кард П. Изв. АН ЭССР, Физ. Матем., 27, № 3, 379-381 (1978).

3. Кард П. Изв. АН ЭССР, Физ. Матем., 29, № 1, 1-7 (1980).

4. Кард П. Изв. АН ЭССР, Физ. Матем., 29, № 2, 113-119 (1980).

5. Кард П. Изв. АН ЭССР, Физ. Матем., 29, № 3, 336-338 (1980).

6. Кард П. Изв. АН ЭССР, Физ. Матем., 30, № 2, 93-101 (1981).

1. Кард П. Изв. АН ЭССР, Физ. Матем., 31, № 3, 241-248 (1982).

Тартуский государственный университет
Поступила в редакцию 4/III 83

\section{P. KARD}

\section{LOPLIKUL KUJUL LAHENDUVAD OHEMÕTMELISED LAINEVORRANDID}

Asendus (9) teisendab võrrandi (8) lainevõrrandiks (16), kus $\Psi_{1}$ on vọ̃rrandi (13) lahend. Parameeter $G$ on seotud koordinaadiga $z$ valemi (17) kaudu, kus $\Psi_{2}$ on võrrandi (13) teine lahend, kusjuures mõlemaid seob valem (14). Lainevorrrandi (16) lahendi (18) saamiseks piisab võrrandi (8) lahendi tundmisest, sest $\Psi=\lim \Theta$. Sel meetodil tuletatakse vōrranditest (19)-(22) lähtudes lainevōrrandid (28), (31), (38), (41), (47), (50), (57), (63), (76), (80)-(82) ja (89), mille lahendid on vastavalt (29), (33), (39), (42), (48), (51), (58), (64), (77), (83)-(85) ja (90); esimese kaheksa puhul annavad parameetri $G$ ja koordinaadi $z$ vahelise seose valemid $(27),(32),(40),(43)$, (49), (52), (56) ja (62); võrrandite $(80)-(82)$ puhul on parameeter $\omega$ seotud koordinaadiga $z$ valemi $(78)$ kaudu. Võrrandid $(76)$ ja $(89)$ on ilma parameetrita.

\section{P. KARD}

\section{ONE-DIMENSIONAL WAVE EQUATIONS WITH CLOSED SOLUTIONS}

The substitution (9) transforms the equation (8) into the wave equation (16), where $\Psi_{1}$ is a solution of the equation (13). The parameter $G$ is connected with the co-ordinate $z$ via the formula (17), where $\Psi_{2}$ is another solution of the equation (13), both satisfying the condition (14). To become the solution (18) of the wave equation (16) only the solution of the equation (8) is needed, as $\Psi=\lim _{k \rightarrow 0} \Theta$. This method is applied to the equations (19)-(22). As a result, thirteen wave equations are obtained, (28), (31), (38), (41), (47), (50), (57), (63), (76), (80)-(82), and (89) whose solutions are (29), (33), (39), (42), (48), (51), (58), (64), (77), (83)-(85), and (90), respectively. In the first eight cases the co-ordinate $z$ is connected with the parameter $G$ via the formulae (27), (32), (40), (43), (49), (52), (56), and $(62)$, respectively. In three cases (equations $(80)-(82))$ another parameter $\omega$ is connected with the co-ordinate via the formula (78). Two equations, (76) and (89), are without parameter. 